\title{
Standardising care of patients requiring non-invasive ventilation in response to NCEPOD - a chief registrar quality improvement project
}

\author{
Authors: Sunit Raja* and Catherine Thomas
}

\section{Introduction}

In 2017, a nationwide review was performed by the National Confidential Enquiry into Patient Outcome and Death (NCEPOD) into the quality of care provided to patients requiring non-invasive ventilation (NIV), in response to rising mortality as measured by the British Thoracic Society (BTS). The report concluded suboptimal clinical or organisational care was provided in over $80 \%$ of cases.

This quality improvement project aimed to review the quality of care of the same cohort of patients at a district general hospital providing acute NIV in four non-critical care wards, to identify specific factors contributing to suboptimal care and produce a practicable solution.

\section{Materials and methods}

A retrospective case-controlled review of 20 case notes of adult patients requiring acute NIV outside of the critical care setting between August to November 2017 by a single respiratory specialist reviewer. Comparison was made against BTS guidance. Outcomes were in-hospital mortality, readmission within 30 days and time to resolution of respiratory acidosis. The review followed the patient journey from acute admission and initiation of NIV through to resolution of acidosis and conclusion of the NIV episode.

\section{Results and discussion}

The local review revealed mortality of $35 \%$ and of the 13 patients that survived the hospital admission, three were re-admitted within 30 days.

There was wide variation in the indication for initiating NIV, with almost all patients treated for ventilatory failure. Pneumonia was correctly identified in $45 \%$ of patients and $15 \%$ patients were ventilated for hypoventilation due to drugs and sedation. The average $\mathrm{pH}$ at initiation of NIV was 7.22 .

Remediable factors were identified along the entire pathway of acute NIV provision including controlled oxygen use, promptness of blood gas sampling, adequate non-ventilator treatment and adequate duration of pre-ventilator treatment. Ventilator settings were titrated inconsistently and $45 \%$ patients did not reach target pressures at all.

\section{Conclusion}

In response to the findings of the review, a protocol was developed to ensure standardised management of patients requiring acute NIV in the first 24 hours. The protocol encompasses indications for initiating NIV, escalation plans in case of treatment failure, blood gas monitoring and titration of pressures. Re-audit is underway to measure improvement in measured outcomes.

\section{Reference}

1 Davidson AC. BTS/ICS guideline for the ventilatory management of acute hypercapnic respiratory failure in adults. Thorax 2016;71(Supp 2):ii1-35. 